\title{
718 WTX-124 IS A NOVEL IL-2 PRO-DRUG THAT IS CONDITIONALLY ACTIVATED IN TUMORS AND DRIVES ANTITUMOR IMMUNITY IN MURINE SYNGENEIC CANCER MODELS
}

Christopher Nirschl* ${ }^{*}$ Heather Brodkin, Daniel Hicklin, Nesreen Ismail, Kristin Morris, Andres Salmeron, Cindy Seidel-Dugan, Philipp Steiner, Zoe Steuert, Jenna Sullivan, William Winston. Werewolf Therapeutics, Cambridge, MA, United States

Background Cancer immunotherapy has established itself as the fourth pillar of cancer treatment thanks to the clinical success of checkpoint inhibitors. However, durable anti-tumor responses following immunotherapy are still limited to certain cancer types, and only a fraction of patients respond to the treatment, demonstrating the need for additional immunotherapeutic agents. Preclinical and clinical studies have demonstrated the promise of cytokine therapy to increase antitumor immunity. One of these key cytokines, interleukin-2 (IL-2), is approved for clinical use in metastatic melanoma and renal cell carcinoma. Unfortunately, this treatment requires dosing every 8 hours due to its poor pharmacokinetic profile and is linked to serious toxicities which limits its utility.

Methods To address these limitations, we designed WTX-124, an IL-2 pro-drug (IL-2 INDUKINE ${ }^{\mathrm{TM}}$ protein) that takes advantage of dysregulated protease activity in the tumor microenvironment (TME) to selectively deliver active IL-2 to the tumor after systemic administration. Peripheral inactivation is achieved by linking the cytokine to an inactivation domain using a tumor protease-sensitive linker. The INDUKINE ${ }^{\mathrm{TM}}$ molecule is also engineered with a half-life extension element to improve the pharmacokinetic profile and maintain longer exposure in the tumor. Once the IL-2 INDUKINE ${ }^{\mathrm{TM}}$ protein reaches the tumor, tumor-associated proteases cleave the linker and release the fully active cytokine.

Results Treatment with WTX-124 resulted in the complete rejection of MC38 tumors in a manner dependent on proteolytic processing of the INDUKINE ${ }^{\mathrm{TM}}$ protein, as a non-cleavable version of WTX 124 lacked anti-tumor activity. Furthermore, the INDUKINE ${ }^{\mathrm{TM}}$ format greatly enhanced the PK profile of WTX 124 over free IL-2, resulting in greater exposure with less overall dosing, and demonstrating a favorable accumulation of free IL-2 in tumors compared to plasma. Mechanistically, WTX 124 treatment drove the expansion and activation of tumor infiltrating CD8 $+\mathrm{T}$ cells and NK cells which increased the production of effector cytokines in the tumor. This resulted in tumor rejection that translated into durable immune memory, as animals that rejected the tumors upon IL-2 INDUKINE ${ }^{\text {TM }}$ protein treatment were protected against a subsequent re-challenge with the same tumor cells. WTX 124 also demonstrated favorable pharmacokinetic and tolerability characteristics in cynomolgus monkeys resulting in minimal release of IL-2 in the periphery.

Conclusions The combination of tumor-selective activation of WTX-124 with reduced peripheral toxicities and its favorable pharmacokinetic profile supports moving this compound into clinical development.

Ethics Approval All animal in vivo work was performed in accordance with current regulations and standards of the U.S. Department of Agriculture and the NIH.

http://dx.doi.org/10.1136/jitc-2021-SITC2021.718 